\title{
The association between serum testosterone and insulin resistance: a longitudinal study
}

\author{
Kristin Ottarsdottir ${ }^{1}$, Anna G Nilsson ${ }^{2,3}$, Margareta Hellgren', Ulf Lindblad' ${ }^{1}$ and Bledar Daka' \\ ${ }^{1}$ Department of Public Health and Community Medicine, Institute of Medicine, Sahlgrenska Academy, University of Gothenburg, Gothenburg, Sweden \\ ${ }^{2}$ Department of Internal Medicine, Institute of Medicine, Sahlgrenska Academy, University of Gothenburg, Gothenburg, Sweden \\ ${ }^{3}$ Department of Endocrinology, Sahlgrenska University Hospital, Gothenburg, Sweden \\ Correspondence should be addressed to K Ottarsdottir: kristin.ottarsdottir@vgregion.se
}

\begin{abstract}
The objective of this study was to investigate whether there is a bidirectional association between testosterone concentrations and insulin resistance, in a prospective population study. A random population sample of 1400 men, aged 30-74, was examined in 2002-2005 in southwestern Sweden and followed up in 2012-2014 ( $N=657)$. After excluding subjects without information on sex hormones and insulin resistance, 1282 men were included in the baseline study. Fasting measurements of plasma glucose, insulin and hormones were performed. Insulin resistance was defined using HOMA-Ir. Mean age at baseline was $47.3 \pm 11.4$ years. From the follow-up survey 546 men were included, mean age $57.7 \pm 11.6$ years. Low concentrations of total testosterone at baseline were significantly associated with high logHOMA-Ir at follow-up in a multivariable model including age, waist-hip ratio, physical activity, alcohol intake, smoking, LDL, CRP, hypertension, diabetes and logHOMA-Ir at baseline as covariates $(\beta=-0.096, P=0.006)$. Similar results were observed for bioavailable testosterone. Men within the lowest quartile of total testosterone at baseline had significantly higher logHOMA-Ir at follow-up than other quartiles (Q1 vs Q2 $P=0.008$, Q1 vs Q3 $P=0.001, \mathrm{Q} 1$ vs $\mathrm{Q} 4 P=0.052$ ). Multivariable analysis of the impact of insulin resistance at baseline on testosterone levels at follow-up revealed no significant associations regarding testosterone concentrations $(\beta=-0.003, P=0.928)$ or bioavailable testosterone ( $\beta=-0.006, P=0.873$ ), when adjusting for baseline concentrations of total testosterone, age, waist-hip-ratio, LDL, CRP, physical activity, alcohol intake, smoking, hypertension and diabetes. Low testosterone concentrations at baseline predicted higher insulin resistance at follow-up, but high insulin resistance at baseline could not predict low testosterone at follow-up.
\end{abstract}

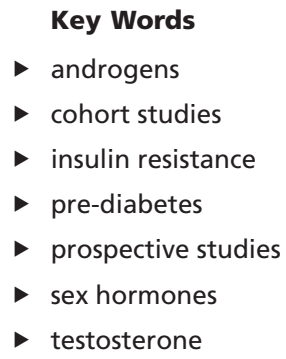

Endocrine Connections (2018) 7, 1491-1500

\section{Introduction}

Testosterone plays a key role in the regulation of glucose and lipid metabolism in men (1) and women (2) although in different manners. Low concentrations of testosterone in men are associated with obesity (3), the metabolic syndrome (4) and hypertension (5). However, it is not fully understood whether there is a causal association or if low testosterone is a surrogate biomarker of poor health (6).
Increased visceral fat leads to raised secretion of proinflammatory cytokines, leptin, estradiol and insulin. These substances may inhibit the activity of the hypothalamic-pituitary gonadal axis at multiple levels, thus lowering the concentrations of testosterone (7). There is also evidence that obesity increases aromatase activity in the fat tissue, converting testosterone to estradiol (8) https://ec.bioscientifica.com https://doi.org/10.1530/EC-18-0480 (c) 2018 The authors Published by Bioscientifica Ltd
This work is licensed under a Creative Commons Attribution-NonCommercial-NoDerivatives 4.0 International License. 
which may play an important role in the regulation of body fat as well (9), and further inhibit testosterone production by negative feedback on the hypothalamic-pituitary gonadal axis (10). On the other hand, low testosterone levels increase the accumulation of visceral body fat, which increases insulin resistance. This is illustrated by the fact that body fat increases in men with prostate cancer undergoing treatment resulting in hypogonadism (11). Furthermore, there is evidence that testosterone could increase insulin sensitivity by decreasing the levels of cytokines (TNFa and IL6), which are believed to increase insulin resistance $(12,13)$. Therefore, it has been suggested that there is a bidirectional relationship between low levels of testosterone and abdominal obesity in men $(7,14,15,16)$, but there is a lack of longitudinal studies investigating this bidirectional relationship.

There are several prospective studies that support the hypothesis that low testosterone levels in men increase the risk for developing diabetes and the metabolic syndrome. Both the NHANES III-study and the Massachusetts Male aging study found an increased risk of type 2 diabetes and the metabolic syndrome in men with low concentrations of testosterone $(17,18,19)$. This association has also been found in older men in the Rancho Bernardo cohort (20) and in a Finnish cohort of middle-aged men (21). Another study investigated levels of sex hormones and the risk of developing type 2 diabetes in a prospective design with 9 -year follow-up (22). The study showed that higher levels of testosterone predicted a lower risk of type 2 diabetes in elderly men. Furthermore, in a recent study of over 2000 community-dwelling middle-aged to elderly men, and with a 5-year follow-up, similar results were found - low total testosterone concentrations predicted the development of type 2 diabetes (23). However, another prospective study including over 5000 men and with a follow-up of 29 years, identified that low testosterone and low levels of SHBG, but not luteinizing hormone $(\mathrm{LH})$, were associated with a higher risk of developing type 2 diabetes, indicating that low testosterone is a risk marker rather than a risk factor for type 2 diabetes, since hypogonadism per se did not seem to be associated with an elevated risk of type 2 diabetes (24). Testosterone has also been shown to decrease insulin resistance, total cholesterol and LDL cholesterol, as shown in one of the largest RCTs investigating the effect of testosterone replacement on insulin resistance in men with type 2 diabetes or metabolic syndrome (25).

While the evidence that low testosterone levels are associated with the development of insulin-resistant states and type 2 diabetes is abundant, less is known about whether insulin resistance influences the decrease in testosterone levels. A bidirectional association has been suggested but the evidence in support of this assumption is weak. Thus, the aim of this study was to investigate whether there is a bidirectional association between testosterone and insulin resistance.

\section{Subjects and methods}

\section{Study population}

This is a longitudinal observational study of the VaraSkövde cohort. Between 2002 and 2005 a sample of 1400 men, aged 30-74, living in southwestern Sweden were randomly selected for a cohort study with the goal to detect risk factors for cardiovascular disease at an early stage (26). During the follow-up visit, a representative sample of the participants was consecutively summoned in 2012-2014, and 657 men completed the study protocol accordingly. Among the invited individuals, 217 men declined participation (details shown in Fig. 1). A nonresponse analysis showed that the non-responders were significantly older (mean difference 2.0 0.4 years, $P<0.001)$, and proportionately more of them were smokers, compared to responders $(21.1 \%$ vs $14.1 \%$, $P<0.001)$, but no other significant differences were found between the groups.

\section{Physical examination}

Specially trained nurses assessed study participants, measuring body weight, height, waist circumference and blood pressure in supine and sitting position at follow-up, supine and standing at baseline. Waist-hip ratio was calculated. Validated questionnaires were used to obtain information on lifestyle including smoking, alcohol intake and leisure time physical activity.

\section{Laboratory analyses}

Fasting venous blood samples were drawn in the morning and after an oral glucose tolerance test. Serum concentrations of testosterone and sex hormone-binding globulin were obtained using radioimmunoassay at baseline and at follow-up (27). All blood samples were immediately frozen at $-82^{\circ} \mathrm{C}$. Diabetes and hypertension were defined based on WHO and JNC8 recommendations, respectively, according to information obtained from medical history and clinical assessment $(28,29)$. Concentrations of insulin and glucose at fasting 


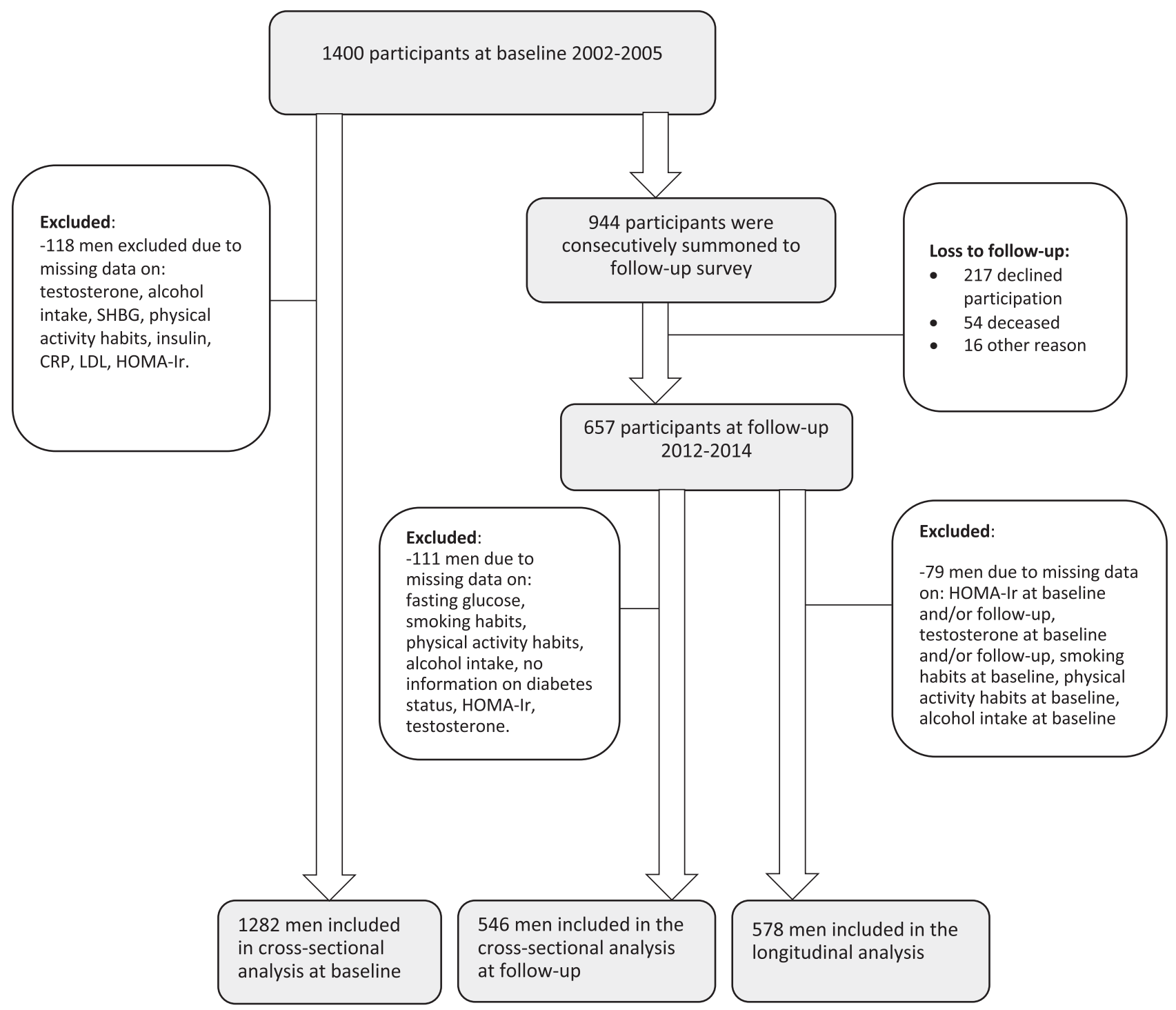

Figure 1

Flow chart showing the study population.

were measured, and HOMA-Ir (homeostatic model assessment of insulin resistance) was calculated by using the formula: fasting insulin $\times$ fasting glucose)/22.5, in subjects without insulin therapy (30). Bioavailable testosterone (which is the sum of free testosterone and the albumin bound testosterone) was calculated using the formula according to Vermeulen et al. (31). Due to changes in insulin measurement methodology (from Roche Cobas to DxI Beckman) in 2011, the insulin values at follow-up were about 35\% higher than at baseline. The insulin values therefore had to be re-calculated with the following formula: New method $=1.3544 \times$ old method +0.3237 . The correlation between methods was $r^{2}=0.9974$. The correlation between methods was investigated within the laboratory which provided the formula for the re-calculation. The method used for analyzing testosterone was radioimmunoassay (RIA) at both visits. However, during the follow-up time, there was a shift on the reagent used in Sweden and Europe from Access Testosterone assay Beckman-Coulter to Elecsys Testosterone II Assay from Roche Diagnostics. The new method gives higher values in total testosterone concentrations compared to the old one used at baseline. We did not have access to an algorithm to adjust for the new method. Due to these changes in testosterone measurement method, analyses investigating change of testosterone over time were not possible. However, strong correlation between the baseline levels of testosterone and follow-up levels was observed (Pearson correlation $0.617, P<0.001)$.

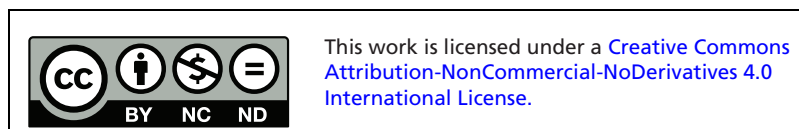


At baseline, the analyses of testosterone were successful in $97.4 \%$ of the participants (1364/1400 individuals). The measurements of insulin were successful in $99.5 \%$ (1393/1400 individuals).

\section{Statistical analyses}

Descriptive statistics were used to describe the characteristics of the study population at baseline and follow-up. Due to skewness in HOMA-Ir, this variable was log-transformed. Theoretical models adjusting for possible confounding factors were built. Linear regression cross-sectional analyses were conducted to investigate the association between sex hormones and insulin resistance at baseline and at follow-up, with logHOMA-Ir as the dependent variable and sex hormone (either total testosterone or bioavailable testosterone) as explanatory variable. The analyses were adjusted for potential confounders, including age, smoking, physical activity, alcohol intake, WHR, LDL, CRP, hypertension and type 2 diabetes mellitus. The longitudinal analyses were conducted using logHOMA-Ir and sex hormones, respectively, at follow-up as dependent variables and sex hormones and $\operatorname{logHOMA-Ir,~respectively,~at~baseline~as~the~}$ explanatory variables. The analyses were adjusted for the same confounders as in the cross-sectional analyses but included also adjustment for the baseline measurement of the outcome variable. General linear models were also performed to compare the variance of log-transformed HOMA-Ir in different quartiles of testosterone. The comparison was pairwise and the lowest quartile was the reference. To investigate age-related differences in the association between bioavailable testosterone and insulin resistance, stratified analyses were conducted for subjects above and below 50 years of age at baseline. Analyses were conducted using IBM SPSS Statistics, version 24 .

\section{Ethics}

The Regional Ethical Review Board in Gothenburg, Sweden, approved the study (D-nr 036-12 and 199-01) and all participants gave their written consent to participation.

\section{Results}

The mean follow-up time was $9.7 \pm 1.4$ years. After excluding subjects with no information in sex hormones and insulin resistance, 1282 men were included in the baseline study. All participants with valid values in all variables used as covariates in the model were included (baseline $N=1282$, follow-up $N=546$, longitudinal analysis $N=578$ ) (Fig. 1). The mean age at baseline was 47.3 years (s.D. 11.4), and at follow-up the mean age was 57.7 years (S.D. 11.6). Further characteristics of the study population at baseline and follow-up are presented in Table 1. In the cross-sectional analyses, concentrations of total serum testosterone were strongly associated with insulin resistance, both at baseline $(\beta=-0.302, P<0.001$, $N=1282)$, and at follow-up $(\beta=-0.325, P<0.001, N=546)$ (Table 2) when adjusted for age. The association was still significant after adjustments for additional relevant confounding factors; waist-hip-ratio, smoking, leisure time physical activity, alcohol intake, LDL, CRP, diabetes status and hypertension (baseline $\beta=-0.140, P<0.001$, $N=1282$, follow-up $\quad \beta=-0.181, \quad P<0.001, \quad N=546$ ). The association was significant also for bioavailable testosterone at baseline when adjusted for age, physical activity and smoking ( $\beta=-0.109, P<0.001, N=1282)$, but not significant when adjusted for waist-hip ratio.

No significant differences in these associations were observed when we stratified for age above and under 50 (excluding adjustment for age) (age $<50 \beta=-0.020$, $P<0.502$; age $\geq 50 \quad \beta=-0.005, P=0.914)$. However, at follow-up, the cross-sectional analysis (not stratified for age) showed a strong and significant inverse association between bioavailable testosterone concentrations and HOMA-Ir even in the fully adjusted model $(\beta=-0.114$, $P=0.004, N=546$ ) (Table 2).

In the longitudinal analysis, low concentrations of total testosterone at baseline were associated with higher levels of $\operatorname{logHOMA-Ir}$ at the follow-up in a multivariable model including age, waist-hip-ratio, baseline logHOMA-Ir, smoking, physical activity, alcohol intake, LDL, CRP, diabetes and hypertension as covariates $\beta=-0.096$, $P=0.006, N=578)$. The longitudinal association was also significant for bioavailable testosterone $(\beta=-0.079$, $P=0.035$ ) (Table 3).

Men within the lowest quartile of total testosterone at baseline had significantly higher HOMA-Ir at follow-up than men within the second and third quartiles. (Mean HOMA-Ir Q1: 2.23, Q2: 1.84, Q3: 1.71, Q4: 1.93. Q1 vs Q2 $\Delta$ mean $=P=0.008, \mathrm{Q} 1$ vs $\mathrm{Q} 3 \Delta$ mean $=P=0.001$ ) whereas the difference was borderline significant between first and fourth quartile (Q1 vs Q4 $\Delta$ mean $=P=0.052$ ), in the fully adjusted model. No significant differences were observed between quartiles 2, 3 and 4 in this regard (Fig. 2).

In the longitudinal analyses investigating the association of insulin resistance at baseline and total or bioavailable testosterone concentrations at follow-up, 
Table 1 Characteristics of the study population.

\begin{tabular}{|c|c|c|}
\hline & Baseline $(N=1282)$ & Follow-up $(N=546)$ \\
\hline Age (years) & $47.3 \pm 11.4$ & $57.7 \pm 11.6$ \\
\hline $\mathrm{BMI}\left(\mathrm{kg} / \mathrm{m}^{2}\right)$ & $26.8 \pm 3.5$ & $27.4 \pm 3.6$ \\
\hline Waist-hip-ratio & $0.94 \pm 0.06$ & $0.97 \pm 0.06$ \\
\hline Systolic blood pressure $(\mathrm{mmHg})$ & $124 \pm 16$ & $126 \pm 13$ \\
\hline Diastolic blood pressure $(\mathrm{mmHg})$ & $72 \pm 10$ & $73 \pm 9$ \\
\hline Fasting insulin $(\mu \mathrm{U} / \mathrm{mL})$ & $6.55 \pm 4.8$ & $7.24 \pm 4.88$ \\
\hline Fasting glucose (mmol/L) & $5.5 \pm 0.85$ & $5.8 \pm 0.9$ \\
\hline HOMA-Ir & $1.7 \pm 1.53$ & $1.9 \pm 1.5$ \\
\hline Serum testosterone $(\mathrm{nmol} / \mathrm{L})$ & $14.3 \pm 4.3$ & $16.0 \pm 5.7$ \\
\hline Current smoker $N(\%)$ & $181(14.1)$ & $52(9.5)$ \\
\hline Hypertension $N(\%)$ & $177(13.8)$ & 79 (14.5) \\
\hline Diabetes mellitus $N(\%)$ & 56 (4.4) & $34(6.2)$ \\
\hline \multicolumn{3}{|l|}{ Level of leisure time physical activity $N(\%)$} \\
\hline Sedentary & $95(7.4)$ & $62(11.4)$ \\
\hline Low level of physical activity & $677(52.8)$ & $287(52.6)$ \\
\hline Moderate level of physical activity & $457(35.6)$ & $163(29.9)$ \\
\hline Strenuous physical activity & $53(4.1)$ & $34(6.2)$ \\
\hline Sex hormone-binding globuline ( $\mathrm{nmol} / \mathrm{L}$ ) & $32.3 \pm 13.3$ & $46.1 \pm 19.6$ \\
\hline
\end{tabular}

Note that the method of measuring serum testosterone changed during the survey time, resulting in higher values at follow-up (described in detail in the 'Methods' section).

a crude significant association was found for total testosterone $(\beta=-0.164, \quad P<0.001)$ and bioavailable testosterone $(\beta=-0.089, \quad P=0.032)$. However, the significance disappeared after adjustment for baseline testosterone/bioavailable testosterone. When stepwise adjustments were made, no significant associations were found in any model in the multivariable analysis ( $\beta=-0.034, P=0.309$ ) (Table 4).

\section{Discussion}

In this prospective cohort study, we aimed to investigate the possible bidirectional relationship between testosterone and insulin resistance by analyzing if a low level of testosterone could predict the development of insulin resistance and vice versa. Our results revealed a significant longitudinal association between lower levels of total testosterone and bioavailable testosterone, respectively, and the development of insulin resistance, which remained significant even after adjustment for possible confounding factors, including waist-hip ratio. This indicates that testosterone itself might have an important and independent effect on the development of insulin resistance over time. However, in this study, the level of insulin resistance at baseline could not predict the decrease in testosterone or bioavailable testosterone level at follow-up. Furthermore, we observed a strong inverse

Table 2 Results from cross-sectional regression analyses showing the association between insulin resistance measured as IgHOMA-Ir and sex hormones (total testosterone and bioavailable testosterone), at baseline and follow-up, respectively.

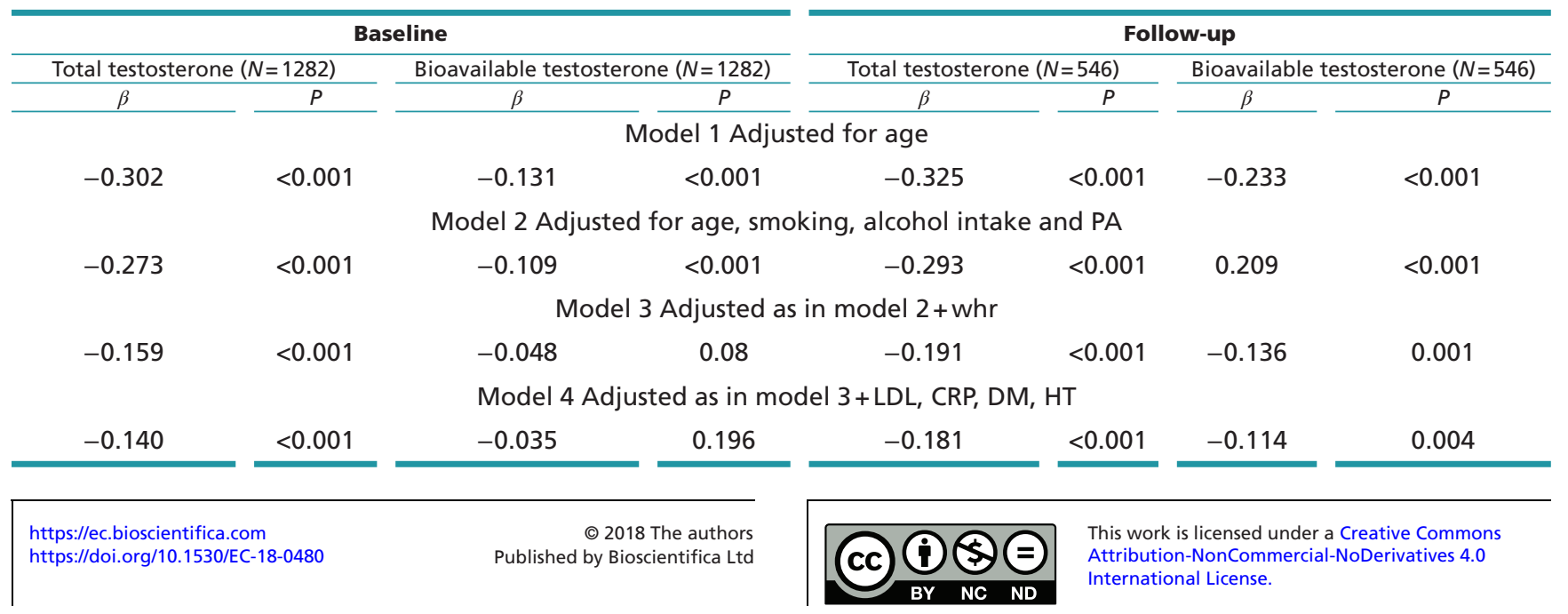


Table 3 Results from longitudinal analyses showing the association between insulin resistance measured as IgHOMA-Ir at follow-up and sex hormones (total testosterone and bioavailable testosterone) at baseline.

\begin{tabular}{|c|c|c|c|}
\hline \multicolumn{2}{|c|}{ Total serum testosterone $(N=578)$} & \multicolumn{2}{|c|}{ Bioavailable testosterone $(N=578)$} \\
\hline$\beta$ & $P$ & $\beta$ & $P$ \\
\hline \multicolumn{4}{|c|}{ Model 1 Adjusted for age and baseline IgHOMA-Ir } \\
\hline-0.147 & $<0.001$ & -0.114 & 0.003 \\
\hline \multicolumn{4}{|c|}{ Model 2 Adjusted for age, baseline IgHOMA-Ir, smoking, alcohol intake and PA } \\
\hline-0.140 & $<0.001$ & -0.109 & 0.005 \\
\hline \multicolumn{4}{|c|}{ Model 3 Adjusted as in model $2+$ whr } \\
\hline-0.102 & 0.004 & -0.083 & 0.027 \\
\hline \multicolumn{4}{|c|}{ Model 4 Adjusted as in model $3+\mathrm{LDL}, \mathrm{CRP}, \mathrm{DM}, \mathrm{HT}$} \\
\hline-0.096 & 0.006 & -0.079 & 0.035 \\
\hline
\end{tabular}

Dependent variable: log-transformed HOMA-Ir.

CRP, c-reactive protein; DM, diabetes mellitus; HT, hypertension; LDL, low density lipoprotein; PA, physical activity; Whr, waist-hip-ratio.

association between concentrations of testosterone and insulin resistance in the cross-sectional analyses at baseline and follow-up.

Although insulin resistance is driven by obesity, there is evidence that suggests that testosterone modulates the expression of the glucose transporter Glut4 and the insulin receptor in muscle and adipose tissue, thereby improving glucose metabolism $(32,33,34)$. The presence of this pathway indicates an effect of testosterone on insulin resistance that is independent from body composition. Furthermore, there is evidence that testosterone withdrawal during 2 weeks in men with idiopathic hypogonadotrophic hypogonadism decreases insulin sensitivity and raises HOMA-Ir without changing BMI or leptin levels (35), suggesting an effect of testosterone on insulin resistance not dependent on change in body weight.

However, in a review from 2011 (7), the association between testosterone and insulin resistance seemed to disappear when adjustments for body composition were added to the models. This tendency can also be seen in our study, where the correlations weakened both in the cross-sectional and in the longitudinal studies when adjustments for waist-hip ratio were added to the linear regression models. This connection between body composition and low testosterone can be explained by different mechanisms. There is evidence that testosterone affects the differentiation of the pluripotent stem cells into the myogenic lineage and inhibits their differentiation into adipocytes (36), thereby influencing the body composition and decreasing insulin resistance. These experimental studies are also supported by randomized controlled studies that show significant beneficial changes in the body composition and insulin resistance in individuals treated with testosterone $(37,38)$.
A reverse causality between testosterone and insulin resistance has been suggested, as in the prospective study by Laaksonen et al. (39). The authors of that study paper did not report an association between insulin resistance and testosterone concentrations, but showed that the metabolic syndrome predicts low testosterone in men in an 11-year follow-up. Moreover, in the same study, the insulin levels at baseline did not predict change in testosterone levels. These results are in line with our findings, suggesting that body composition but not insulin resistance is associated in a bidirectional manner with testosterone levels. The impact of body composition on testosterone is further supported by observations in

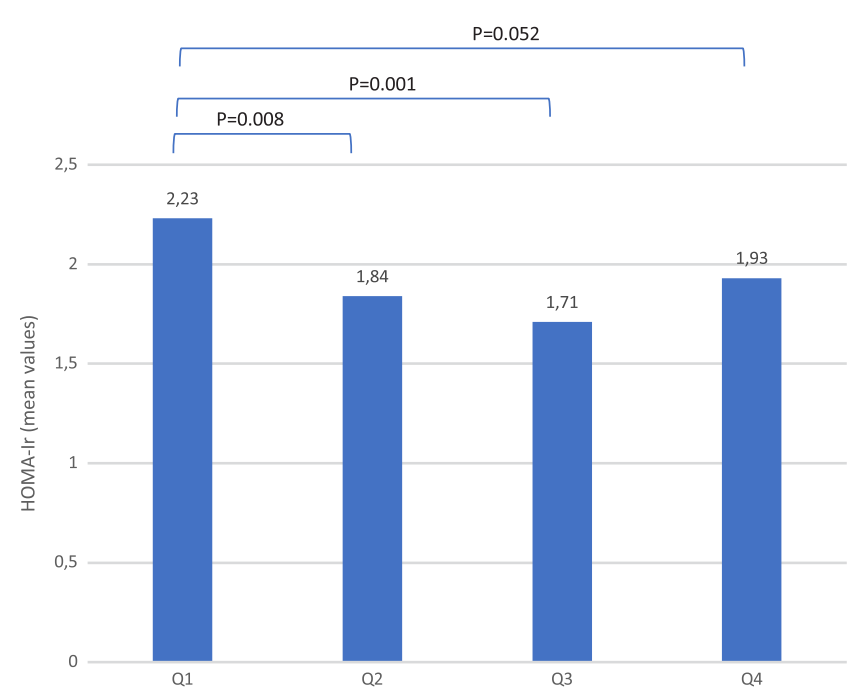

Figure 2

Quartiles of serum testosterone in follow-up survey. Men with the lowest testosterone concentration at baseline (Q1) had the highest HOMA-Ir at follow-up. Model adjusted for age, waist-hip ratio and HOMA-Ir at baseline. 
Table 4 Results from longitudinal regression analyses showing the association between insulin resistance measured as IgHOMA-Ir at baseline and sex hormones (total testosterone and bioavailable testosterone) at follow-up.

\begin{tabular}{|c|c|c|c|}
\hline \multicolumn{2}{|c|}{ Total serum testosterone $(N=578)$} & \multicolumn{2}{|c|}{ Bioavailable testosterone $(N=578)$} \\
\hline$\beta$ & $P$ & $\beta$ & $P$ \\
\hline \multicolumn{4}{|c|}{ Model 1 Adjusted for age and baseline sex hormone level } \\
\hline-0.034 & 0.309 & -0.066 & 0.052 \\
\hline \multicolumn{4}{|c|}{ Model 2 Adjusted as in model $1+$ smoking, alcohol intake and PA } \\
\hline-0.024 & 0.479 & -0.054 & 0.119 \\
\hline \multicolumn{4}{|c|}{ Model 3 Adjusted as in model $2+$ whr } \\
\hline 0.013 & 0.727 & -0.001 & 0.987 \\
\hline \multicolumn{4}{|c|}{ Model 4 Adjusted as in model 3+LDL, CRP, DM, HT } \\
\hline 0.003 & 0.928 & -0.006 & 0.873 \\
\hline
\end{tabular}

CRP, c-reactive protein; DM, diabetes mellitus; HT, hypertension; LDL, low density lipoprotein; PA, physical activity; Whr, waist-hip-ratio.

individuals undergoing gastric bypass surgery where testosterone levels increased significantly following the dramatic weight loss $(40,41)$. In accordance with these observations, it has also been shown that weight gain or diabetes type 2 drive the decline in testosterone levels over time $(42,43)$. There are models explaining the impact of body composition on testosterone concentrations, as stated in the hypogonadal-obesityadipocytokine hypothesis $(8,9,10,44)$. This shows that aromatase activity in adipose tissue converts testosterone to estradiol, which in turn inhibits the hypothalamicpituitary-gonadal axis, leading to decreased testosterone levels. Leptin and adipocytokines, such as TNFa and IL6, produced in the fat tissue further inhibit the hypothalamic-pituitary-gonadal axis. In addition, leptin also has a negative effect on the Leydig cells, by inhibiting the stimulation of gonadotropin, leading to even further decreased testosterone level. However, even though obesity is associated with higher inflammatory activity, inflammation measured as CRP did not influence the association between testosterone and insulin resistance in our study. Although the evidence is strong that body composition influences testosterone concentrations and insulin resistance, there is also evidence that testosterone has a short-acting effect on insulin resistance, which cannot be explained by change in body weight or body composition, as discussed above (35). However, most of the associations seen in observational studies seem to be explainable by the body composition. Our findings also support the lack of an independent effect of insulin resistance on testosterone levels, as no significant association between insulin resistance at baseline and testosterone levels in follow-up were found. The suggested bidirectional relationship was thus not confirmed. This could be due to the existence of differences that

https://ec.bioscientifica.com
https://doi.org/10.1530/EC-18-0480

were too small to be detectable with the use of this method or due to small sample size and long follow-up time. However, even though there may be a bidirectional association, the direction where insulin resistance decreases testosterone levels is much weaker than in the other direction.

In our cross-sectional analyses, there was a strong association between both total testosterone and bioavailable testosterone and insulin resistance at baseline and at follow-up. These results are consistent with other cross-sectional studies $(45,46,47)$. However, in the analyses of the bioavailable testosterone, the significance was lost at baseline after adjustments for confounding. There are several possible explanations for the differences in these associations. First, the participants in the study were of different ages at these timepoints, suggesting that the testosterone level is more important for insulin resistance in elderly men. The loss of significance when adjusting for waist-hip ratio indicates that body composition, and visceral obesity in particular, might have a stronger impact on the insulin resistance progress in younger individuals. However, when age-stratified analyses were conducted for subjects above and below 50 years of age at baseline, similar results were obtained in the two groups; significance was lost when waist-hip ratio was added to the adjustment model. This does not support the existence of age differences in these associations. However, there seems to be a non-linear association between testosterone at baseline and HOMA-Ir at follow-up (Fig. 2). In fact, only the lowest quartile differed significantly from the other quartiles, suggesting a threshold effect. In the follow-up survey, more participants may have reached below that threshold, due to higher age and higher prevalence of abdominal obesity, thus showing a stronger and significant association.

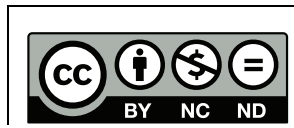

This work is licensed under a Creative Commons Attribution-NonCommercial-NoDerivatives 4.0 International License. 
Second, different methods of measuring testosterone were used in the baseline survey and in the follow-up, as discussed below. As the method was more precise at follow-up, the associations might be stronger and still significant after adjustments for possible confounding.

\section{Strengths and limitations}

The strength of this study is its longitudinal design with high participation rates at both baseline and follow-up visits. Moreover, the large sample size and the systematic characterization of the study population along with the long follow-up time are obvious strengths in this study. The extensive information on the participants enabled us to account for various covariates in the analyses. Due to the size of the study, these results should be valid in other male Caucasian populations.

Some limitations should be mentioned, however. First, the methods of analysing insulin and serum testosterone changed during the observation period, and, therefore, it was not possible to study the change of testosterone or HOMA-Ir over time. Such change in the analyses of the variables is a limitation of the study and may affect the results to some extent due to lower precision and power, which could result in a type 2 error. However, the laboratory did internal validity testing and found high correlation between the new and old method. Thus, these measurements of insulin should be considered comparable, even though we did not do any analyses including change or delta value.

Regarding testosterone it was not possible to compare the levels and to estimate the changes due to the change in the method. Therefore, we were not able to analyse factors that influence the change in testosterone. However, the information about testosterone at baseline permitted adjustments and thus, an estimation of the correlation between insulin at baseline and testosterone at follow-up.

Second, measurements of testosterone were made with radioimmunoassay, which is considered less reliable than mass spectrometry. However, this is particularly true for low concentrations, such as in women or children, and less problematic in healthy adult men with higher concentrations. The radioimmunoassays used showed an acceptable correlation with mass spectrometry values $(48,49)$. Also, radioimmunoassay is generally used in clinical practice. Due to less reliability when using radioimmunoassay, there is a risk of lower precision in the analyses. However, even with this lower precision we observed a strong association, probably owing to the large size of the cohort. Regarding the measurements of insulin, we only had one fasting value, taken at the same time in all participants. Since insulin is a pulsatile hormone, it would have been an advantage to calculate the mean value from repeated measurements in order to reduce the risk of type 2 error in the analyses due to lower precision. However this was not possible due to the large sample size, and furthermore, when calculating HOMA-Ir, a single measurement has been considered an acceptable compromise, that obtains similar results in large sample sizes (50). We chose to adjust for waist-hip-ratio to see whether the association between testosterone and insulin resistance was independent of body composition. This might have caused a type 2 error, especially in the crosssectional analyses at baseline because of collinearity. However, one of the questions we wanted to investigate was whether testosterone influenced insulin resistance through other pathways than the body composition. Thus, we chose to adjust the analyses even for waist-hip-ratio.

Despite the mentioned limitations, there was a consistency in the results, both in the cross-sectional and the longitudinal analyses.

\section{Conclusion}

We observed a robust association between low testosterone concentrations and insulin resistance, both crosssectionally and longitudinally, but this relationship was not bidirectional. While low concentrations of testosterone independently predicted the increase in insulin resistance, no significant impact of insulin resistance on testosterone concentrations was observed during the follow-up time. Further studies including sex hormone-binding globulin and estradiol may provide more insight into the role of sex hormones on the deterioration of insulin resistance.

\section{Declaration of interest}

The authors declare that there is no conflict of interest that could be perceived as prejudicing the impartiality of the research reported.

\section{Funding}

The study was funded by The Local Research and Development Council Göteborg och Södra Bohuslän, Agreement concerning research and education of doctors, The health care committee in Region Västra Götaland and University of Gothenburg.

\section{Acknowledgements}

The authors would like to thank the participants from Vara and Skövde who made this study possible. 


\section{References}

1 Jones TH. Testosterone deficiency: a risk factor for cardiovascular disease? Trends in Endocrinology and Metabolism 201021 496-503. (https://doi.org/10.1016/j.tem.2010.03.002)

2 Morford J \& Mauvais-Jarvis F. Sex differences in the effects of androgens acting in the central nervous system on metabolism. Dialogues in Clinical Neuroscience 201618 415-424.

3 Mah PM \& Wittert GA. Obesity and testicular function. Molecular and Cellular Endocrinology 2010316 180-186. (https://doi.org/10.1016/j. mce.2009.06.007)

4 Brand JS, Rovers MM, Yeap BB, Schneider HJ, Tuomainen TP, Haring R, Corona G, Onat A, Maggio M, Bouchard C, et al. Testosterone, sex hormone-binding globulin and the metabolic syndrome in men: an individual participant data meta-analysis of observational studies. PLOS ONE 20149 e100409. (https://doi. org/10.1371/journal.pone.0100409)

5 Svartberg J, von Muhlen D, Schirmer H, Barrett-Connor E, Sundfjord J \& Jorde R. Association of endogenous testosterone with blood pressure and left ventricular mass in men. The Tromso Study. European Journal of Endocrinology 2004150 65-71. (https://doi. org/10.1530/eje.0.1500065)

6 Shores MM \& Matsumoto AM. Testosterone, aging and survival: biomarker or deficiency. Current Opinion in Endocrinology, Diabetes, and Obesity 201421 209-216. (https://doi.org/10.1097/ MED.0000000000000057)

7 Grossmann M. Low testosterone in men with type 2 diabetes: significance and treatment. Journal of Clinical Endocrinology and Metabolism 201196 2341-2353. (https://doi.org/10.1210/jc.20110118)

8 Cohen PG. Aromatase, adiposity, aging and disease. The hypogonadal-metabolic-atherogenic-disease and aging connection. Medical Hypotheses 200156 702-708. (https://doi.org/10.1054/ mehy.2000.1169)

9 Finkelstein JS, Lee H, Burnett-Bowie S-AM, Pallais JC, Yu EW, Borges LF, Jones BF, Barry CV, Wulczyn KE, Thomas BJ, et al. Gonadal steroids and body composition, strength, and sexual function in men. New England Journal of Medicine 2013369 1011-1022. (https:// doi.org/10.1056/NEJMoa1206168)

10 Cohen PG. The hypogonadal-obesity cycle: role of aromatase in modulating the testosterone-estradiol shunt - a major factor in the genesis of morbid obesity. Medical Hypotheses 1999 52 49-51. (https://doi.org/10.1054/mehy.1997.0624)

11 Smith MR, Finkelstein JS, McGovern FJ, Zietman AL, Fallon MA, Schoenfeld DA \& Kantoff PW. Changes in body composition during androgen deprivation therapy for prostate cancer. Journal of Clinical Endocrinology and Metabolism 200287 599-603. (https://doi. org/10.1210/jcem.87.2.8299)

12 Malkin CJ, Pugh PJ, Jones RD, Kapoor D, Channer KS \& Jones TH. The effect of testosterone replacement on endogenous inflammatory cytokines and lipid profiles in hypogonadal men. Journal of Clinical Endocrinology and Metabolism 200489 3313-3318. (https://doi. org/10.1210/jc.2003-031069)

13 Hotamisligil GS. Inflammatory pathways and insulin action. International Journal of Obesity and Related Metabolic Disorders 200327 (Supplement 3) S53-S55. (https://doi.org/10.1038/sj.ijo.0802502)

14 Grossmann M. Diagnosis and treatment of hypogonadism in older men: proceed with caution. Asian Journal of Andrology 201012 783-786. (https://doi.org/10.1038/aja.2010.107)

15 Grossmann M, Gianatti EJ \& Zajac JD. Testosterone and type 2 diabetes. Current Opinion in Endocrinology, Diabetes and Obesity 2010 17 247-256. (https://doi.org/10.1097/MED.0b013e32833919cf)

16 Traish A, Saad F, Feeley R \& Guay A. The dark side of testosterone deficiency: III. Cardiovascular disease. Journal of Andrology 200930 477-494. (https://doi.org/10.2164/jandrol.108.007245)
17 Kupelian V, Page ST, Araujo AB, Travison TG, Bremner WJ \& McKinlay JB. Low sex hormone-binding globulin, total testosterone, and symptomatic androgen deficiency are associated with development of the metabolic syndrome in nonobese men. Journal of Clinical Endocrinology and Metabolism 200691 843-850. (https://doi. org/10.1210/jc.2005-1326)

18 Selvin E, Feinleib M, Zhang L, Rohrmann S, Rifai N, Nelson WG, Dobs A, Basaria S, Golden SH \& Platz EA. Androgens and diabetes in men: results from the Third National Health and Nutrition Examination Survey (NHANES III). Diabetes Care 200730 234-238. (https://doi.org/10.2337/dc06-1579)

19 Stellato RK, Feldman HA, Hamdy O, Horton ES \& McKinlay JB. Testosterone, sex hormone-binding globulin, and the development of type 2 diabetes in middle-aged men: prospective results from the Massachusetts male aging study. Diabetes Care 200023 490-494. (https://doi.org/10.2337/diacare.23.4.490)

20 Oh JY, Barrett-Connor E, Wedick NM \& Wingard DL. Endogenous sex hormones and the development of type 2 diabetes in older men and women: the Rancho Bernardo study. Diabetes Care 200225 55-60. (https://doi.org/10.2337/diacare.25.1.55)

21 Laaksonen DE, Niskanen L, Punnonen K, Nyyssönen K, Tuomainen T-P, Valkonen V-P, Salonen R \& Salonen JT. Testosterone and sex hormone-binding globulin predict the metabolic syndrome and diabetes in middle-aged men. Diabetes Care 200427 1036-1041. (https://doi.org/10.2337/diacare.27.5.1036)

22 Salminen M, Vahlberg T, Raiha I, Niskanen L, Kivela SL \& Irjala K. Sex hormones and the risk of type 2 diabetes mellitus: a 9-year follow up among elderly men in Finland. Geriatrics and Gerontology International 201515 559-564. (https://doi.org/10.1111/ ggi.12312)

23 Gyawali P, Martin SA, Heilbronn LK, Vincent AD, Taylor AW, Adams RJT, O'Loughlin PD \& Wittert GA. The role of sex hormonebinding globulin (SHBG), testosterone, and other sex steroids, on the development of type 2 diabetes in a cohort of community-dwelling middle-aged to elderly men. Acta Diabetologica 201855 861-872. (https://doi.org/10.1007/s00592-018-1163-6)

24 Holmboe SA, Jensen TK, Linneberg A, Scheike T, Thuesen BH, Skakkebaek NE, Juul A \& Andersson AM. Low testosterone: a risk marker rather than a risk factor for type 2 diabetes. Journal of Clinical Endocrinology and Metabolism 2016101 3180-3190. (https://doi. org/10.1210/jc.2016-1778)

25 Jones TH, Arver S, Behre HM, Buvat J, Meuleman E, Moncada I, Morales AM, Volterrani M, Yellowlees A, Howell JD, et al. Testosterone replacement in hypogonadal men with type 2 diabetes and/or metabolic syndrome (the TIMES2 study). Diabetes Care 2011 34 828-837. (https://doi.org/10.2337/dc10-1233)

26 Larsson CA, Daka B, Gullberg B, Rastam L \& Lindblad U. Clusters of AMI risk factors and their association with left ventricular hypertrophy: a population-based study within the Skaraborg Project, Sweden. International Journal of Cardiology 2013168 5416-5421. (https://doi.org/10.1016/j.ijcard.2013.08.047)

27 Kamal S. Radioimmunoassay and related techniques. Journal of the Pakistan Medical Association 199343 224-225.

28 World Health Organization. Definition, Diagnosis and Classification of Diabetes Mellitus and its Complications. Part 1: Diagnosis and Classification of DIabetes Mellitus WHO/NCD/NCS/99, 2nd ed. Geneva, Switzerland: World Health Organization, 1999.

29 World Health Organization. A Global Brief on Hypertension - Silent Killer, Global Public Health Crisis. Geneva, Switzerland: World Health Organization, 2013. (available at: http://www.who.int/ cardiovascular_diseases/publications/global_brief_hypertension/en/)

30 Matthews DR, Hosker JP, Rudenski AS, Naylor BA, Treacher DF \& Turner RC. Homeostasis model assessment: insulin resistance and beta-cell function from fasting plasma glucose and insulin concentrations in man. Diabetologia 198528 412-419. (https://doi. org/10.1007/BF00280883)

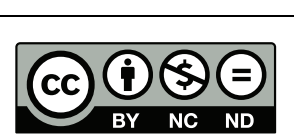

This work is licensed under a Creative Commons Attribution-NonCommercial-NoDerivatives 4.0 International License. 
31 Vermeulen A, Verdonck L \& Kaufman JM. A critical evaluation of simple methods for the estimation of free testosterone in serum. Journal of Clinical Endocrinology and Metabolism 199984 3666-3672. (https://doi.org/10.1210/jcem.84.10.6079)

32 Chen X, Li X, Huang HY, Li X \& Lin JF. Effects of testosterone on insulin receptor substrate- 1 and glucose transporter 4 expression in cells sensitive to insulin. Zhonghua Yi Xue Za Zhi 200686 1474-1477.

33 Sato K, Iemitsu M, Aizawa K \& Ajisaka R. Testosterone and DHEA activate the glucose metabolism-related signaling pathway in skeletal muscle. American Journal of Physiology: Endocrinology and Metabolism 2008294 E961-E968. (https://doi.org/10.1152/ajpendo.00678.2007)

34 Muthusamy T, Murugesan P \& Balasubramanian K. Sex steroids deficiency impairs glucose transporter 4 expression and its translocation through defective Akt phosphorylation in target tissues of adult male rat. Metabolism: Clinical and Experimental 200958 1581-1592. (https://doi.org/10.1016/j.metabol.2009.05.010)

35 Yialamas MA, Dwyer AA, Hanley E, Lee H, Pitteloud N \& Hayes FJ. Acute sex steroid withdrawal reduces insulin sensitivity in healthy men with idiopathic hypogonadotropic hypogonadism. Journal of Clinical Endocrinology and Metabolism 200792 4254-4259. (https:// doi.org/10.1210/jc.2007-0454)

36 Singh R, Artaza JN, Taylor WE, Gonzalez-Cadavid NF \& Bhasin S. Androgens stimulate myogenic differentiation and inhibit adipogenesis in $\mathrm{C} 3 \mathrm{H}$ 10T1/2 pluripotent cells through an androgen receptor-mediated pathway. Endocrinology 2003144 5081-5088. (https://doi.org/10.1210/en.2003-0741)

37 Kapoor D, Goodwin E, Channer KS \& Jones TH. Testosterone replacement therapy improves insulin resistance, glycaemic control, visceral adiposity and hypercholesterolaemia in hypogonadal men with type 2 diabetes. European Journal of Endocrinology $2006 \mathbf{1 5 4}$ 899-906. (https://doi.org/10.1530/eje.1.02166)

38 Dhindsa S, Ghanim H, Batra M, Kuhadiya ND, Abuaysheh S, Sandhu S, Green K, Makdissi A, Hejna J, Chaudhuri A, et al. Insulin resistance and inflammation in hypogonadotropic hypogonadism and their reduction after testosterone replacement in men with type 2 diabetes. Diabetes Care 201639 82-91. (https://doi.org/10.2337/dc15-1518)

39 Laaksonen DE, Niskanen L, Punnonen K, Nyyssonen K, Tuomainen TP, Valkonen VP \& Salonen JT. The metabolic syndrome and smoking in relation to hypogonadism in middle-aged men: a prospective cohort study. Journal of Clinical Endocrinology and Metabolism 200590 712-719. (https://doi.org/10.1210/jc.2004-0970)

40 Hammoud A, Gibson M, Hunt SC, Adams TD, Carrell DT, Kolotkin RL \& Meikle AW. Effect of Roux-en-Y gastric bypass surgery on the sex steroids and quality of life in obese men. Journal of Clinical Endocrinology and Metabolism 200994 1329-1332. (https://doi. org/10.1210/jc.2008-1598)
41 Reis LO, Favaro WJ, Barreiro GC, de Oliveira LC, Chaim EA, Fregonesi A \& Ferreira U. Erectile dysfunction and hormonal imbalance in morbidly obese male is reversed after gastric bypass surgery: a prospective randomized controlled trial. International Journal of Andrology 201033 736-744. (https://doi.org/10.1111/ j.1365-2605.2009.01017.x)

42 Travison TG, Araujo AB, Kupelian V, O'Donnell AB \& McKinlay JB. The relative contributions of aging, health, and lifestyle factors to serum testosterone decline in men. Journal of Clinical Endocrinology and Metabolism 200792 549-555. (https://doi.org/10.1210/jc.20061859)

43 Haring R, Ittermann T, Volzke H, Krebs A, Zygmunt M, Felix SB, Grabe HJ, Nauck M \& Wallaschofski H. Prevalence, incidence and risk factors of testosterone deficiency in a population-based cohort of men: results from the study of health in Pomerania. Aging Male 201013 247-257. (https://doi.org/10.3109/13685538. 2010.487553)

44 Kelly DM \& Jones TH. Testosterone: a metabolic hormone in health and disease. Journal of Endocrinology 2013217 R25-R45. (https://doi. org/10.1530/JOE-12-0455)

45 Tajar A, Huhtaniemi IT, O'Neill TW, Finn JD, Pye SR, Lee DM, Bartfai G, Boonen S, Casanueva FF, Forti G, et al. Characteristics of androgen deficiency in late-onset hypogonadism: results from the European Male Aging Study (EMAS). Journal of Clinical Endocrinology and Metabolism 201297 1508-1516. (https://doi.org/10.1210/ jc.2011-2513)

46 Grossmann M. Testosterone and glucose metabolism in men: current concepts and controversies. Journal of Endocrinology 2014220 R37-R55. (https://doi.org/10.1530/JOE-13-0393)

47 Grossmann M, Thomas MC, Panagiotopoulos S, Sharpe K, Macisaac RJ, Clarke S, Zajac JD \& Jerums G. Low testosterone levels are common and associated with insulin resistance in men with diabetes. Journal of Clinical Endocrinology and Metabolism 200893 1834-1840. (https://doi.org/10.1210/jc.2007-2177)

48 Wang C, Catlin DH, Demers LM, Starcevic B \& Swerdloff RS Measurement of total serum testosterone in adult men: comparison of current laboratory methods versus liquid chromatography-tandem mass spectrometry. Journal of Clinical Endocrinology and Metabolism 200489 534-543. (https://doi.org/10.1210/jc.2003-031287)

49 Dittadi R, Matteucci M, Meneghetti E \& Ndreu R. Reassessment of the Access Testosterone chemiluminescence assay and comparison with LC-MS method. Journal of Clinical Laboratory Analysis 201832 e22286. (https://doi.org/10.1002/jcla.22286)

50 Wallace TM, Levy JC \& Matthews DR. Use and abuse of HOMA modeling. Diabetes Care 200427 1487-1495. (https://doi. org/10.2337/diacare.27.6.1487)

Received in final form 10 November 2018

Accepted 4 December 2018

Accepted Preprint published online 4 December 2018
This work is licensed under a Creative Commons Attribution-NonCommercial-NoDerivatives 4.0 International License. 